\title{
Sinopsis de la familia Acanthaceae en el Perú
}

\author{
A synopsis of the family Acanthaceae in Peru
}

Rosa M. Villanueva-Espinoza ${ }^{1, *}$ y Florangel M. Condo ${ }^{1}$

Recibido: 03 marzo 2019 | Aceptado: 28 abril 2019 | Publicado en línea: 30 junio 2019 Citación: Villanueva-Espinoza, RM; Condo, FM. 2019. Sinopsis de la familia Acanthaceae en el Perú. Revista Forestal del Perú 34(1): 21-40. DOI: http://dx.doi.org/10.21704/rfp.v34i1.1282

\begin{abstract}
Resumen
La familia Acanthaceae en el Perú solo ha sido revisada por Brako y Zarucchi en 1993, desde entonces, se ha generado nueva información sobre esta familia. El presente trabajo es una sinopsis de la familia Acanthaceae donde cuatro subfamilias (incluyendo Avicennioideae) y 38 géneros son reconocidos. El tratamiento de cada género incluye su distribución geográfica, número de especies, endemismo y carácteres diagnósticos. Un total de ocho nombres (Juruasia Lindau, Lophostachys Pohl, Teliostachya Nees, Streblacanthus Kuntze, Blechum P. Browne, Habracanthus Nees, Cylindrosolenium Lindau, Hansteinia Oerst.) son subordinados como sinónimos y, tres especies endémicas son adicionadas para el país.
\end{abstract}

Palabras clave: Acanthaceae, actualización, morfología, Perú, taxonomía

\begin{abstract}
The family Acanthaceae in Peru has just been reviewed by Brako and Zarruchi in 1993, since then, new information about this family has been generated. The present work is a synopsis of family Acanthaceae where four subfamilies (includying Avicennioideae) and 38 genera are recognized. Treatment of each genus includes description, geographical distribution, number of species, endemism and diagnostic characters. A total of eight names (Juruasia Lindau, Lophostachys Pohl, Teliostachya Nees, Streblacanthus Kuntze, Blechum P. Browne, Habracanthus Nees, Cylindrosolenium Lindau, Hansteinia Oerst) are subordinated as synonyms and, three endemics species are added to the country.
\end{abstract}

Key words: Acanthaceae, update, morphology, Peru, taxonomy

${ }^{1}$ División de Ecología Vegetal - CORBIDI, Lima, Perú.

* Autor de Correspondencia: rosvillanuevae@gmail.com 


\section{Introducción}

La familia Acanthaceae está representada por especies con flores conspicuas y un atractivo follaje como Acanthus mollis L., Aphelandra aurantiaca (Scheidw.) Lindl. y Sanchezia speciosa Leonard, ampliamente solicitadas en los invernaderos y presentando un alto valor ornamental (Leonard 1951, Durkee 1986). Además, Acanthaceae cumple un importante rol ecológico al poseer un amplio rango de adaptaciones ecológicas y formas florales relacionadas con una gran diversidad de síndromes de polinización y cambios de polinizador (Schmidt-Lebuhn et al. 2007, Daniel y Lott 2016).

Las Acanthaceae, con aproximadamente 4750 especies y 190 géneros, es una de las 12 familias de angiospermas más diversas en el mundo (Tripp y Fatimah 2012, Daniel y McDade 2014). A lo largo del Neotrópico, Acanthaceae posee una gran riqueza de especies siendo México y Centro América, las porciones mejores inventariadas. Asimismo, América del Sur es considerada como una zona rica en especies, principalmente la parte norte de Argentina, Venezuela, Ecuador, Bolivia, Colombia y Perú (Tripp y Luján 2018).

En el Perú, la familia Acanthaceae carece de una revisión general al no ser incluida en Flora of Perú (MacBride 1936-1962). Sin embargo, en el Catálogo de Angiospermas y Gimnospermas del Perú (Brako y Zarucchi 1993) se reportaron 262 especies y 39 géneros de Acanthaceae para el país. Desde entonces, producto de revisiones taxonómicas de esta familia en países aledaños (Leonard 1951, Wasshausen y Wood 2004, Wasshausen 2013a), tratamientos recientes de géneros Neotropicales (Ezcurra 1993, 2002; Schmidt-Lebuhn 2003; Wasshausen y Wood 2004; Daniel y McDade 2014; Kameyama 2008; Côrtes 2013; Tripp y Koenemann 2015; Daniel 2016) y exploraciones en el Perú (Wasshausen 1996, 1997a, 2006, 2013b) se han reportado nuevos registros y especies que han ido contribuyendo al conocimiento taxonómico de la familia. Sin embargo, no existe una literatura con los datos actualizados de la familia Acanthaceae para el país. Por ello, el objetivo principal de este trabajo es presentar un panorama general de la familia Acanthaceae en el Perú, incluyendo su última clasificación y el número actualizado de géneros y especies. Asimismo, proporcionar datos sobre la distribución, el número de especies endémicas y caracteres diagnósticos de los géneros peruanos.

\section{Historia Taxonómica}

A lo largo de la historia, la familia Acanthaceae ha sufrido una serie de cambios con respecto a su posición taxonómica. A nivel inter-familiar, Acanthaceae ha sido ubicada en diferentes órdenes, los primeros botánicos consideraron a Acanthaceae dentro del Orden Tubiflorae junto con Solanaceae, Bignoniaceae, entre otros (Lindau 1895, Engler y Diels 1936, Melchior 1964). En base a la corola zigomórfica, reducción progresiva del número de estambres y filotaxis opuesta, Hutchinson (1973) incluyó a Acanthaceae en el Orden Personales, sin embargo, fue ubicada en el Orden Scrophulariales bajo el sistema de Takhtajan (1987). Cronquist (1988) mantuvo a Acanthaceae en este orden, diferenciándola de Scrophulariaceae por la dehiscencia exclusiva de los frutos y funículo especializado. Posteriormente, Thorne (1992) incluyó a Acanthaceae en el Orden Bignoniales colocando a Acanthaceae en el suborden Bignonineae.

En base a datos moleculares, Olmstead et al. (1993) incluyó a Acanthaceae en el orden Lamiales junto a Scrophulariaceae, Callitrichaceae, etc. Otros autores siguieron esta clasificación (Thorne 2007, Takhtajan 2009, Reveal 2012), así como, el sistema de clasificación filogenético de APG. En la actualidad, el APG IV (2016) mantiene a Acanthaceae en el Orden Lamiales, cuyos caracteres diagnósticos son la presencia de hojas opuestas, flores mayormente zigomórficas, tricomas glandulares con células radiales, flavonoides, oligosacáridos cornósidos y verbascósidos (Stevens 2017).

\section{Clasificación Intrafamiliar}

La primera descripción de los géneros de Acanthaceae fue publicada por Linneo (1753a, 1753b) en su clásico "Species Plantarum", entre 
los que destacan Acanthus L., Avicennia L., Dianthera L. y Justicia L. Posteriormente, Jussieu (1789) clasificó a Acanthaceae en dos grupos: los que poseen dos estambres, conformado por los géneros Justicia y Dianthera; y los de cuatro estambres "didínamos", constituido por Acanthus L., Dilivaria Juss., Blepharis Juss., Thunbergia Retz., Barleria L. y Ruellia L. En base a la presencia o ausencia del retináculo, Nees (1847) reconoció dos subórdenes: Anechmatacantheae y Echmatacantheae. Algunas de las tribus de Echmatacantheae se convirtieron en rango de subtribus o géneros dentro de la tribu Justicieae, según la clasificación de Bentham (1876) que reconoció 5 tribus en Acanthaceae. Clarke (1885) siguió la clasificación de Bentham, sin embargo, varió el número de subtribus que conformaban la tribu Ruellieae y Justicieae.

Lindau (1895) en base al tipo de fruto, número de óvulos y presencia/ausencia de retináculo divide a Acanthaceae en cuatro subfamilias: (1) Nelsonioideae, (2) Thumbergioideae, (3) Acanthoideae y (4) Mendoncioideae. Van Tieghem (1908), sustentó que Acanthaceae solo estaba representada por Acanthoideae incluyendo las otras subfamilias en Thunbergiaceae. Sin embargo, Wettstein (1924) consideró estas subfamilias como tribus y las localizó en Thunbergioideae, presentando Acanthaceae dos subfamilias: (1) Acanthoideae y (2) Thunbergioideae. Posteriormente, Bremekamp (1965) excluiría los géneros que carecían de semillas con retináculo reconociendo dos subfamilias: (1) Acanthoideae y (2) Ruellioideae. Cronquist (1988), excluyó el género Nelsonia R. Br. de Scrophulariaceae y junto a Thunbergiaceae, les dió el rango de subfamilia reconociendo en Acanthaceae tres subfamilias: (1) Acanthoideae, (2) Nelsonioideae y (3) Thunbergioideae, siendo Mendoncioideae tratada como una familia separada. Y Thorne (1992) reconoció cinco subfamilias para Acanthaceae: (1) Nelsonioideae, (2) Thunbergioideae, (3) Acanthoideae, (4) Ruellioideae y (5) Mendoncioideae.

En los últimos años, se han realizado numerosos estudios sistemáticos de Acanthaceae, entre ellos destacan los de Scotland et al. (1995) y McDade et al. (2000b) quienes demostraron que Thunbergia, Nelsonioideae y Mendoncia no pueden ser separados de Acanthaceae como lo menciona Bremekamp (1965). Scotland y Vollesen (2000) incluyeron a Mendoncioideae y Ruellioideae en Thunbergioideae y Acanthoideae, respectivamente. Por consiguiente, Acanthaceae solo estuvo conformada por tres subfamilias: (1) Nelsonioideae, (2) Thunbergioideae y (3) Acanthoideae. Asimismo, sugirieron localizar al género Avicennia en la familia Acanthaceae. En base a caracteres morfológicos y datos moleculares, Schwarzbach y McDade (2002) sustentaron la inclusión de Avicennia L. en Acanthaceae y posteriormente, Thorne (2007) dilucidó su posición convirtiéndola en la cuarta subfamilia de Acanthaceae. Actualmente, Acanthaceae está conformada por 4 subfamilias: (1) Thunbergioideae, (2) Nelsonioideae, (3) Acanthoideae y (4) Avicennioideae (Tripp y McDade 2014). En la Figura 1, se grafica los cambios taxonómicos que ha sufrido Acanthaceae.

\section{Morfología}

Acanthaceae se puede distinguir por las hojas opuestas decusadas, nudos hinchados, línea interpeciolar más o menos marcada, presencia de brácteas y/o bractéolas conspicuas, fruto cápsula, y carencia de caracteres organolépticos como olor o látex. La mayoría de especies son de porte herbáceo o arbustivo, pocos son los géneros que presentan especies arbóreas ya sean árboles pequeños (Suessenguthia Merxm., Bravaisia DC. y Trichanthera Kunth) o grandes (Avicennia L.); mientras que Thunbergia Retz. y Mendoncia Vell. ex Vand. son exclusivamente lianas o enredaderas (Gentry 1993, Singh 2004, Wasshausen y Wood 2004).

La subfamilia representativa es Acanthoideae caracterizándose por la presencia del fruto cápsula con dehiscencia explosiva y cistolitos (excepto Acantheae) (McDade et al. 2008). Nelsonioideae está representada por especies con filotaxis alterna, inflorescencias con brácteas alternas y semillas con endospermo; Thunbergioideae está conformada por especies enredaderas que presentan un cáliz reducido, 


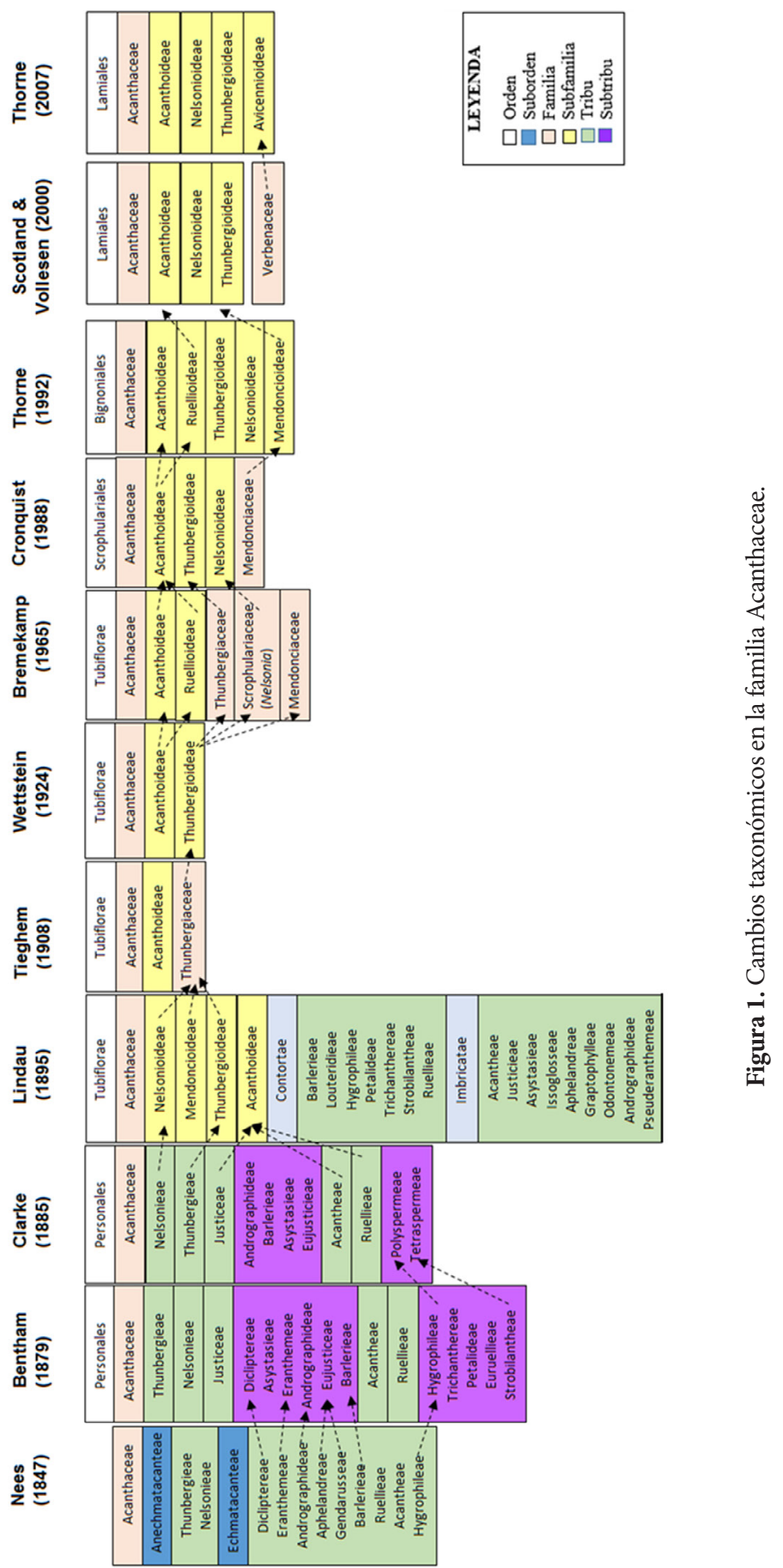




\begin{tabular}{|c|c|c|c|c|}
\hline \multicolumn{2}{|c|}{ Subfamilia y Linajes } & $\begin{array}{c}\mathrm{N}^{0} \text { géneros } \\
\text { Neotropicales }\end{array}$ & Referencias & $\begin{array}{l}\mathrm{N}^{0} \text { géneros } \\
\text { Peruanos }\end{array}$ \\
\hline \multicolumn{2}{|c|}{ Subf. Thunbergioideae } & 1 & Borg et al. (2008) & 1 \\
\hline \multicolumn{2}{|c|}{ Subf. Avicennioideae } & 1 & Daniel (2016) & 1 \\
\hline \multicolumn{2}{|c|}{ Subf. Nelsonioideae } & 3 & McDade et al. ( 2012) & 1 \\
\hline \multicolumn{5}{|c|}{ Subf. Acanthoideae } \\
\hline \multicolumn{2}{|c|}{ Acantheae } & 10 & McDade et al. (2005) & 5 \\
\hline \multirow{3}{*}{$\begin{array}{l}\text { Clado } \\
\text { Cistolitos }\end{array}$} & Justicieae & 36 & $\begin{array}{l}\text { McDade et al. (2000a), Kiel } \\
\text { et al. (2006), Daniel et al. } \\
\text { (2008), Daniel (2009), Kiel } \\
\text { et al. (2017) }\end{array}$ & 15 \\
\hline & Ruellieae & 15 & Tripp et al. (2013) & 9 \\
\hline & $\begin{array}{c}\text { BAWN (Barlerieae, } \\
\text { Andrographideae, } \\
\text { Whitfieldieae y } \\
\text { Neuracanthus) }\end{array}$ & 3 & $\begin{array}{l}\text { Martín-Bravo y Daniel } \\
\qquad(2016)\end{array}$ & 2 \\
\hline \multicolumn{2}{|c|}{ TOTAL } & 69 & - & 34 \\
\hline
\end{tabular}

Cuadro 1. Comparación de los números de géneros de Acanthaceae en el Perú respecto del total de géneros Neotropicales.

dos bractéolas grandes y un fruto drupa o cápsula y, Avicennioideae está constituido por el género Avicennia ("mangles") que debido a al ambiente salino que habita presenta adaptaciones morfológicas y fisiológicas como neumatóforos para la respiración (Daniel y McDade 2014, Daniel 2016).

\section{Número de especies y géneros}

La familia Acanthaceae comprende aproximadamente 4750 especies y 190 géneros (Daniel y McDade 2014), ampliamente distribuidas en las regiones tropicales y subtropicales del mundo con centros de mayor endemismo, diversidad morfológica y riqueza de especies en el Viejo Mundo (Indomalasia, África incluyendo Madagascar) y en el Nuevo Mundo (Sudamérica, Centroamérica y México) (Daniel y Acosta 2003). Pese a ser considerada una familia pantropical, solo 14 géneros son considerados como tales, Avicennia L., Barleria
L., Dicliptera Juss., Dyschoriste Nees, Elytraria Michx., Hygrophila R. Br., Justicia L., Lepidagathis Wild., Mendoncia Vell. ex Vand., Nelsonia R. Br., Oplonia Raf., Pseuderanthemum Radlk., Ruellia L. y Staurogyne Wall. (Tripp y McDade 2014, Martín-Bravo y Daniel 2016). Su distribución en el Nuevo Mundo se detalla en el Cuadro 1. Aproximadamente, se han registrado alrededor de 69 géneros nativos de esta región, el $50 \%$ de ellos están presentes en el Perú. Braz y Monteiro (2017) mencionan a Perú como zona de distribución del género Staurogyne, sin embargo, no se lista ningún espécimen para el país. Por ello, en el presente trabajo se prefiere no considerarlo como un género peruano.

En el Perú, Brako y Zarucchi (1993) registraron 262 especies en 39 géneros. Luego, Ulloa Ulloa et al. (2004), en base a material de herbario y literatura publicada, adicionaron 15 especies, entre nuevos registros, nuevas especies y cambios nomenclaturales. En los últimos 


\begin{tabular}{|c|c|}
\hline Brako y Zarucchi (1993) & Género actualizado \\
\hline Juruasia Lindau & Herpetacanthus Nees \\
\hline $\begin{array}{c}\text { Lophostachys } \text { Pohl } \\
\text { Teliostachya } \text { Nees }\end{array}$ & $\begin{array}{c}\text { Lepidagathis } \text { Willd. } \\
\text { Streblacanthus Kuntze }\end{array}$ \\
\hline Blechum P. Browne & Puellia L. \\
\hline $\begin{array}{c}\text { Habracanthus } \text { Nees } \\
\text { Cylindrosolenium } \text { Lindau } \\
\text { Hansteinia } \text { Oerst. }\end{array}$ & Stenostephanus Nees \\
\hline
\end{tabular}

Cuadro 2. Nombres de géneros actualizados de Acantáceas peruanas que han cambiado desde el Catálogo de Brako y Zarucchi (1993).

años, se han seguido reportando nuevos cambios taxonómicos y nomenclaturales en esta familia (Wasshausen 1996, 1997b, 1999; Ezcurra 2002; Vásquez et al. 2002; Schmidt-Lebuhn 2003; Wasshausen y Wood 2003, 2004; Wasshausen 2006; Indriunas 2011; Côrtes 2013; Daniel y McDade 2014). Se encontró que Aphelandra caput-medusae Lindau, Aphelandra hartwegiana Nees, Bravaisia integerrima (Spreng.) Standl., Elytraria klugii Leonard, Neriacanthus lehmannianus (Lindau) Lindau y Ruellia macrophylla Vahl especies registradas para el Perú (Leonard 1951, 1958; Wasshausen 1975), no fueron considerados por Brako y Zarucchi (1993). Asimismo, muchos nombres científicos se han reducido a sinónimos o han sufrido algún cambio nomenclatural en tratamientos posteriores (Wasshausen 1996, 1997a, 1999; Wasshausen y Wood 2001; Schwarzbach y McDade 2002; Schmidt-Lebuhn 2003; Wasshausen y Wood 2003, 2004; McDade y Tripp 2007; Wood 2009; Tripp y McDade 2012; Côrtes 2013; Wasshausen 2013a; Tripp y Koenemann 2015). De igual manera, los nombres de algunos géneros peruanos se han sinonimizado (Cuadro 2).

En el Cuadro 3, se muestra los datos numéricos de las especies de cada género en el Perú. Considerando taxones nativos e introducidos, Justicia (64) es el género más rico en especies, seguido de Aphelandra (50) y Sanchezia (48). Los géneros Thunbergia, Hypoestes y Acanthus presentan 1-3 especies, todas introducidas en el país. Además, las especies Megaskepasma erythrochlamys Lindau, Justicia spicigera Schltdl., Justicia brandegeeana Wassh. \& L.B. Sm., Justicia spicigera Schltdl. y Pseuderanthemum carruthersii (Seem.) Guillaumin son ampliamente cultivadas en el Perú. Las especies de Acanthaceae ocupan casi todos los hábitats intertropicales excepto, los de alta montaña (por encima de los $3000 \mathrm{msnm}$ ). Estas especies pueden encontrarse en sabanas, yungas, bosques de tierras bajas, desiertos, selva alta perennifolia, manglares, bosque nublado, lagos y áreas con agua dulce corriente (Daniel y Acosta 2003, Daniel y Lott 2016).

Con respecto a los endemismos del Perú, León (2006) reportó 42 especies y tres (3) géneros endémicos (Cephalacanthus Lindau, Orophochilus Lindau y Trichosanchezia Mildbr) en las regiones Bosque Muy Húmedo Montano y Bosque Húmedo Amazónico entre una altitud de 100 a $2870 \mathrm{msnm}$. En base a recientes publicaciones (Wood 2009, Côrtes 2013, Daniel y McDade 2014), se adicionan 3 especies endémicas para el Perú, Stenostephanus macrolobus (Lindau) J.R.I. Wood, Elytraria klugii Leonard. y Pachystachys linearibracteata A.L.A. Côrtes. La primera fue registrada en el departamento de Huancavelica y las dos últimas, en el departamento de San Martín.

\section{Descripción de los géneros reportados para el Perú}

1. Acanthus L., Sp. Pl. 2: 639 (1753). Tipo: Acanthus mollis L.

Género nativo del Viejo Mundo con alrededor de 8-10 especies, principalmente, arbustos o subarbustos (Wasshausen 2013a). Presenta un amplio rango geográfico incluyendo el Mediterráneo, sur de Europa, África, Asia Meridional, Malesia y Australasia (McDade et al. 2005). En el Perú, solo está registrado Acanthus mollis L., especie introducida y cultivada, común en los jardines del departamento de Lima. Carácter distintivo: Hojas arrosetadas, corola unilabiada, 4 estambres y ausencia de cistolitos. 


\begin{tabular}{|c|c|}
\hline Género & $N^{a}$ Especies \\
\hline Acanthus L. & 1 \\
\hline Aphelandra $\mathrm{R} . \mathrm{Br}$. & 50 \\
\hline Avicennia L. & 1 \\
\hline Barleria L. & 1 \\
\hline Bravaisia DC. & 1 \\
\hline Cephalacanthus Lindau & 1 \\
\hline Dicliptera Juss. & 13 \\
\hline Dyschoriste Nees & 2 \\
\hline Elytraria Michx. & 2 \\
\hline Encephalosphaera Lindau & 1 \\
\hline Fittonia E. Coem. & 2 \\
\hline Herpetacanthus Nees & 2 \\
\hline Hygrophila $\mathrm{R}$. Br. & 1 \\
\hline Hypoestes Soland. ex R. Br. & 1 \\
\hline Justicia L. & 64 \\
\hline Kalbreyeriella Lindau & 2 \\
\hline Lepidagathis Wild. & 4 \\
\hline Megaskepasma Lindau & 1 \\
\hline Mendoncia Vand. & 26 \\
\hline Neriacanthus Benth. & 1 \\
\hline Odontonema Nees & 1 \\
\hline Oplonia Raf. & 2 \\
\hline Orophocilus Lindau & 1 \\
\hline Pachystachys Nees & 16 \\
\hline Pseuderanthemum Radlk. & 7 \\
\hline $\begin{array}{c}\text { Pulchranthus V.M. Baum, Reveal \& } \\
\text { Nowicke }\end{array}$ & 1 \\
\hline Razisea Oerst. & 2 \\
\hline Ruellia L. & 30 \\
\hline Sanchezia Ruiz \& Pav. & 48 \\
\hline Stenandrium Nees & 2 \\
\hline Stenostephanus Nees & 7 \\
\hline Strobilanthes Blume & 1 \\
\hline Suessenguthia Merxm. & 6 \\
\hline Tessmanniacanthus Mildbr. & 1 \\
\hline Tetramerium Nees & 7 \\
\hline Thunbergia Retz & 3 \\
\hline Trichanthera Kunth & 1 \\
\hline Trichosanchezia Mildbr. & 1 \\
\hline
\end{tabular}

2. Aphelandra R. Br., Prodr. 475 (1810). Especie Lectotipo: Aphelandra cristata (Jacq.) R. $\mathrm{Br}$.

Género neotropical conformado por 175 especies de hierbas, arbustos o árboles pequeños. Se distribuye desde el noroeste de México hasta el sureste de Brasil. Su centro de concentración de especies se encuentra en los Andes de Colombia, Ecuador, Perú y Bolivia (Daniel 1991, Wasshausen 2013b). En el Perú, se han registrado 50 especies siendo 5 de estas endémicas (Leonard 1951; Wasshausen 1975; Brako y Zarucchi 1993; Wasshausen 1996; Wasshausen y Wood 2004; León 2006; Wasshausen 2006, 2013b). Carácter distintivo: Corola bilabiada, 4 estambres, anteras monotecas y ausencia de cistolitos (McDade 1984) (ver Figuras 2A, 2I y 3A).

3. Avicennia L., Sp. Pl. 1: 110 (1753). Tipo: Avicennia officinalis L.

Género pantropical conformado por 8 especies de manglares (Tomlinson 1986). Se distribuye en regiones marítimas de los trópicos y subtrópicos de todo el mundo (Daniel 2016). En el Perú, solo está registrada Avicennia germinans (L.) L. "Mangle negro" en el departamento de Tumbes (Brako y Zarucchi 1993). Carácter distintivo: Hojas coriáceas, neumatóforos, hojas con excreción salina, 2 estilos, cápsula coriácea y ausencia de cistolitos.

4. Barleria L., Sp. Pl. 2: 636 (1753). Especie Lectotipo: Barleria cristata L.

Género pantropical conformado por 300 especies de hierbas o arbustos (Balkwill y Balkwill 1997). Se distribuye principalmente en Asia y África, con pocas especies presentes en América (Gibson 1974). En el Perú, esta registrada la especie introducida Barleria cristata L. cultivada en el departamento de Junín (Brako y Zarucchi 1993). Carácter distintivo: Cáliz tetralobado, lobos desiguales, corola pentalobada, brácteas florales espinosas y presencia de cistolitos (Balkwill y Balkwill 1997).

Cuadro 3. Número actualizado de especies presentes en el Perú por cada género. 
5. Bravaisia DC., Biblioth. Universelle Geneve 17: 132-133 (1838). Tipo: Bravaisia floribundia DC.

Género tropical conformado por 3 especies de arbusto o árboles. Se distribuye en México, Cuba, América Central, Colombia, Perú y Venezuela (Durkee 1978, Daniel 1988). En el Perú, solo está registrada la especie nativa Bravaisia integerrima (Spreng.) Standl. (Leonard 1958; Daniel 1988). Carácter distintivo: Corola bilabiada, estambres 4 , tecas mucronadas, presencia de cistolitos y raíces aéreas (Daniel 1988).

6. Cephalacanthus Lindau, Repert. Spec. Nov. Regni Veg. 1: 158 (1905). Tipo: Cephalacanthus maculatus Lindau.

Género monotípico conformado por la especie Cephalacanthus maculatus Lindau, endémico del Perú, distribuyéndose en la cuenca del río Mayo en el departamento de San Martín a 1400 msnm (León 2006). Carácter distintivo: Inflorescencia capituliforme, bráctea redondeada, cáliz pentalobado, corola bilabiada, tubo corto subcilíndrico, filamentos 2 y presencia de cistolitos (Lindau 1905).

7. Dicliptera Juss., Ann. Mus. Natl. Hist. Nat. 9: 267-269 (1807). Tipo: Dicliptera chinensis (L.) Juss. (= Justicia chinensis L.).

Género pantropical con cerca de 175 especies de hierbas o arbustos (Daniel 2009). Se distribuye en América, África, Asia y Australia (Wasshausen 2013a). En el Perú, se han reportado 13 especies en un rango altitudinal entre 0-3600 msnm (Brako y Zarucchi 1993). León (2006) registró a Dicliptera porphyrea Lindau y Dicliptera rauhii Wassh. como especies endémicas para el país, sin embargo, Wasshausen (2004) considera a D. rauhii como sinónimo de Dicliptera squarrosa Nees, especie ampliamente distribuida en América del Sur. Carácter distintivo: Tallo hexagonal, bractéolas florales dispuestas en 2 ciclos o más, estambres 2 , cápsula con placenta que se separa de la pared interna y presencia de cistolitos.

8. Dyschoriste Nees in Wall., Pl. Asiat. Rar. 3: 75, 81 (1832). Especie lectotipo: Dyschoriste depressa Nees.
Género pantropical conformado con alrededor de 80 especies de hierbas o subarbustos (Chumchim et al. 2015). Se distribuye en América, África y Asia, presentando una mayor diversidad en los trópicos de Capricornio y Cáncer en el Nuevo Mundo (Daniel 2004, Wasshausen y Wood 2004). En el Perú, se han registrado 2 especies, Dyschoriste repens (Nees) Kuntze y Dyschoriste quitensis (Kunth) Kuntze. Carácter distintivo: Lobos calicinos fusionados al menos desde la tercera parte de su longitud, margen hialino, corola bilabiada, anteras mucronadas y presencia de cistolitos (Ezcurra 2014).

9. Elytraria Michx., Fl. Bor. Amer. 1: 8 (1803). Tipo: Elytraria virgata Michx.

Género pantropical de aproximadamente 22 especies de hierbas o arbustos. Se distribuye en América, África, Madagascar y Sudeste Asiático (Vollesen 2008, Wenk y Daniel 2009). En el Perú, se ha registrado Elytraria imbricata (Vahl) Pers. y Elytraria klugii Leonard, siendo esta última endémica (Brako y Zarucchi 1993, Daniel y McDade 2014). Carácter distintivo: Hierbas caulescentes o no, pedúnculo y brácteas florales con escamas alternas o en espiral, cáliz tetrapartido con lobo anterior bilobado o bífido, cápsula sin retináculo y ausencia de cistolitos (Wenk y Daniel 2009).

10. Encephalosphaera Lindau., Bull. Herb. Boiss., ser. 2, 4: 322 (1904). Tipo: Encephalosphaera vitellina Lindau.

Género neotropical conformado por 4 especies de hierbas o arbustos. Se distribuye desde Colombia hacia Ecuador y desde el Este de Perú hacia Brasil (Wasshausen 2013a). En el Perú, solo está registrada Encephalosphaera lasiandra Mildbr. para los departamentos de Ayacucho, Cusco, Madre de Dios y San Martín (Brako y Zarucchi 1993). Carácter distintivo: Estambres 4, estaminodio 1, anteras monotecas, polen globoso con superficie rugulada de aspecto cerebriforrne-serpentino y ausencia de cistolitos (Leonard 1953).

11. Fittonia E. Coem., Fl. Des Serres 15:185 (1865). Tipo: Fittonia verschaffeltii 


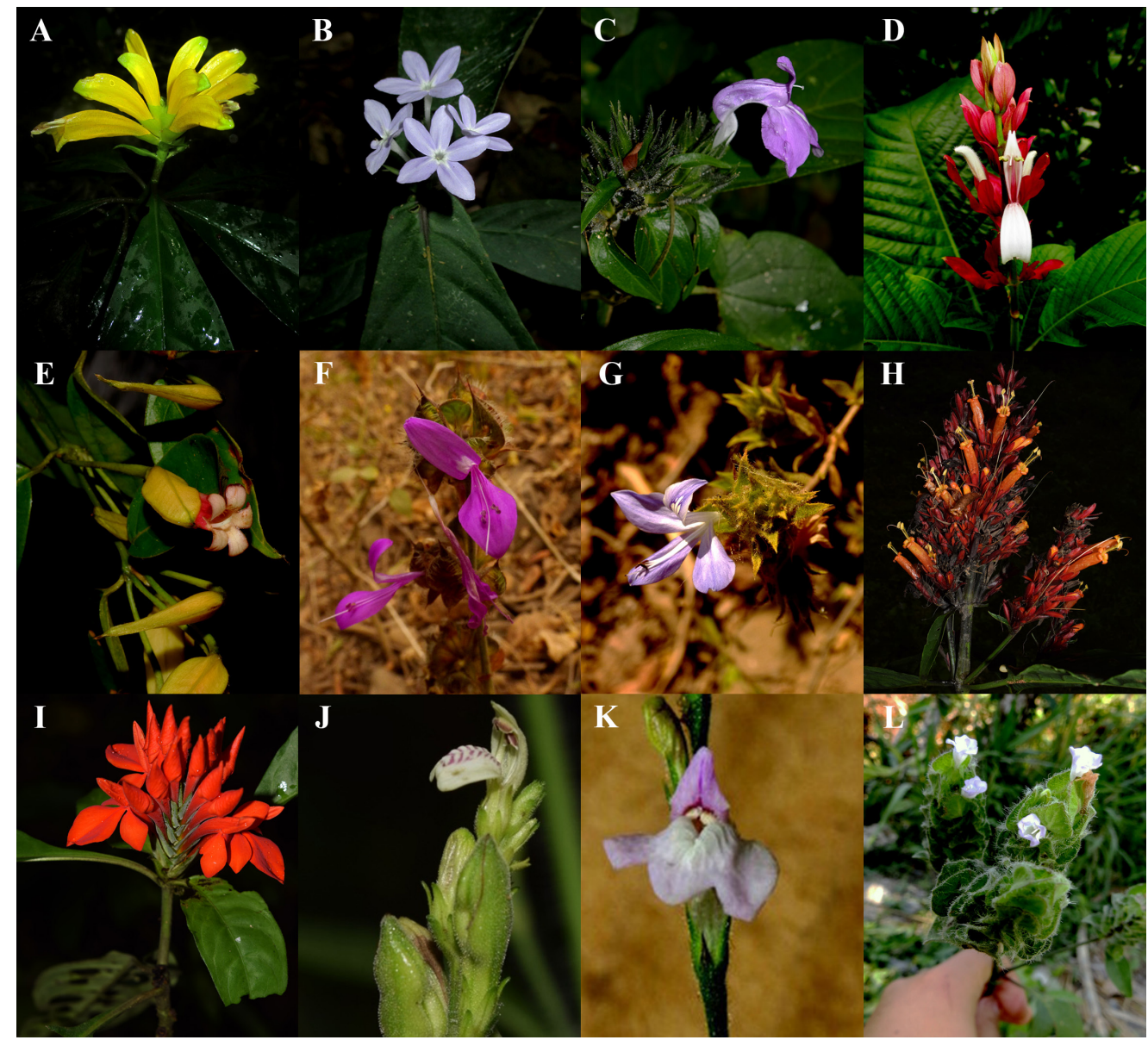

Figura 2. Especies de Acanthaceae presente en el Perú. A. Aphelandra knappiae Wassh.; B. Pseuderanthemum lanceolatum (Ruiz \& Pav.) Wassh.; C. Justicia rusbyi (Lindau) V.A.W. Graham; D. Megaskepasma erythrochlamys Lindau; E. Mendoncia aspera Ruiz \& Pav.; F. Dicliptera peruviana (Lam.) Juss.; G. Tetramerium wasshausenii T.F. Daniel; H. Sanchezia conferta Leonard; I. Aphelandra aurantiaca Lindl.; J. Justicia glutinosa (Bremek.) V.A.W. Graham; K. Justicia ulei Lindau; L. Ruellia blechum L. Fotos por: A-E Robin Fernandez-Hilario; F y G Florangel Condo; H-L Rosa Villanueva.

(Lem.) Van Houtte (= Fittonia albivensis (Lindl. Ex Veitch) Brummit).

Género neotropical conformado por 2 especies herbáceas, nativas de Ecuador, Bolivia y Perú. Se distribuye principalmente en el nor-occidente de Sudamérica hasta Bolivia, siendo cultivado en centroamérica y el sur de Asia (Boggan et al. 1997, Wasshausen y Wood 2004). En el Perú, están registradas Fittonia albivenis (Lindl. ex Veitch) Brummitt y Fitto- nia gigantea Linden (Brako y Zarucchi 1993). Carácter distintivo: Hojas con venación marcadamente colorida, base cordada, corola bilabiada, estambres 2, anteras ditecas y presencia de cistolitos (Wasshausen 2013a) (ver Figura 3C).

12. Herpetacanthus Nees., Pl. Nouv. Amer. 159 (1847). Tipo: Herpetacanthus longiflorus Nees.

Género neotropical conformado por 25 especies de arbustos o subarbustos. Se distribuy- 
en desde Costa Rica hacia Perú y Brasil, donde alcanza su mayor concentración (Indriunas y Kameyama 2012, Wasshausen 2013a). En el Perú dos especies son reconocidas Herpetacanthus rotundatus (Lindau) Bremek. y Herpetacanthus acuminatus (Lindau) Bremek. (Brako y Zarucchi 1993). Carácter distintivo: Corola bilabiada, estambres didínamos, anteras con 1 a 2 tecas y presencia de cistolitos (Wasshausen 2013a).

13. Hygrophila R.Br., Prodr. 479 (1810). Tipo: Hygrophila angustifolia R. Br.

Género pantropical conformado por 100 especies de hierbas acuáticas o higrófilas. En el Perú, se ha registrado una especie Hygrophila costata Nees en un rango altitudinal entre 0 a 1500 msnm (Brako y Zarucchi 1993, Vollesen 2008). Carácter distintivo: Corola bilabiada, estambres 4, a veces, 2 estambres y 2 estaminodios, semillas con tricomas higroscópicos y presencia de cistolitos (Wasshausen 2013a).

14. Hypoestes Soland. ex R. Br., Prodr. 474 (1810). Tipo: Hypoestes floribunda R. Br.

Género paleotropical conformado por 150 especies de hierbas o arbustos. Se distribuye en Asia, Australia, África y las Indias Orientales. Su centro de origen es el Viejo Mundo, siendo cultivado en el Neotrópico (Al-Hakimi et al. 2015, Darbyshire 2015). En el Perú, solo se ha registrado la especie introducida Hypoestes phyllostachya Baker que se encuentra asilvestrada en el departamento de Junín. Carácter distintivo: Hojas con manchas rosadas, brácteas florales (2) conspicuas, corola bilabiada resupinada, anteras monotecas y presencia de cistolitos (ver Figura 3E).

15. Justicia L., Sp. Pl. 1:15 (1753). Tipo: Justicia adhatoda L.

Género cosmopolita conformado por casi 700 especies de hierbas o arbustos, siendo el más grande y complejo de la familia Acanthaceae (Graham 1988, Wasshausen 2013a). Presenta una mayor riqueza en los trópicos y subtrópicos del Nuevo Mundo, extendiéndose a las zonas templadas de Norte América y el Sur de América del Sur (Kiel et al. 2018). En el Perú se han reportado 64 especies siendo 5 especies endémicas (Wasshausen 1997a; Brako y Zarucchi 1993; Ezcurra 2002; Wasshausen y Wood 2003, 2004; León 2006). Carácter distintivo: Corola bilabiada, labio superior con rúgula, estambres 2 , tecas mucronadas a múticas, anteras ditecas y presencia de cistolitos (ver Figura 2C, 2J y 2K).

16. Kalbreyeriella Lindau., Notizbl. Berlin 8: 143 (1922). Tipo: Kalbreyeriella rostellata Lindau.

Género neotropical conformado por 3 especies de hierbas o arbustos. Se distribuye en América Central y Colombia (Leonard 1958). En Perú se han registrado 2 especies, Kalbreyeriella gigas Leonard y Kalbreyeriella rostellata Lindau (Brako y Zarucchi 1993). Carácter distintivo: Corola rostrada (antes de la expansión), color escarlata, estambres 2, exertos, anteras uni- o ditecas y presencia de cistolitos (Wasshausen 2013a).

17. Lepidagathis Willd., Sp. Pl. 3: 400 (1800). Tipo: Lepidagathis cristata Willd

Género paleotropical conformado por más de 100 especies entre hierbas o arbustos (Wasshausen 2013a). Se distribuye principalmente en África, India y Malesia (Wasshausen y Wood 2004). Además, incluye especies americanas debido a que posee como sinónimo al género anteriormente considerado neotropical Lophostachys Pohl (Kameyama 2008). En el Perú, se han reportado 4 especies (Brako y Zarucchi 1993, Wasshausen y Wood 2004, Wasshausen 2006). Carácter distintivo: Cáliz pentalobado, lobos desiguales, corola bilabiada, estambres 4 , anteras ditecas (a veces reducidos a 1 teca) y presencia de cistolitos (Kameyama 2008).

18. Megaskepasma Lindau, Bull. Herb. Boissier 5 (8): 666 (1897). Tipo: Megaskepasma erythrochlamys Lindau.

Género neotropical monotípico conformado por la especie arbustiva Megaskepasma erythrochlamys Lindau (Durkee 1978). Nativo de Venezuela y Colombia, distribuyéndose desde el Sur de México hasta Brasil, extendiéndose a Panamá, Nicaragua, Costa Rica y Ecuador 
debido a su amplio cultivo (Soto y Vega 2010, Wasshausen 2013a). En el Perú, está cultivado y asilvestrado en los departamentos de Lima, Pasco y Junín. Carácter distintivo: Brácteas vistosas de color fucsia, corola bilabiada, estambres 2, tecas múticas y ausencia de cistolitos (Durkee 1986).

19. Mendoncia Vand., Fl. Lusit. Bras. Spec. 43 (1788). Tipo: Mendoncia aspera Ruiz \& Pav.

Género neotropical conformado por alrededor de 90 especies de enredaderas herbáceas o leñosas (Wasshausen 2017). Se distribuye principalmente desde el Sur de México hacia el sur de Brasil, con pocas especies en el Oeste de África y Madagascar (Schönenberger y Endress 1998). En el Perú, están registradas 26 especies, siendo Mendoncia peruviana Leonard y Mendoncia tarapotana Lindau endémicas (Brako y Zarucchi 1993, Vásquez et al. 2002, León 2006). Carácter distintivo: Tallo con xilema disperso, bractéolas (2) cubriendo la corola, cáliz pequeño, fruto drupáceo y ausencia de cistolitos (Wasshausen 2017).

20. Neriacanthus Benth. in Benth \& Hook, Gen. Pl. 2: 1096 (1876). Tipo: Neriacanthus purdieanus Benth.

Género neotropical conformado por 5 especies de hierbas o arbustos. Se distribuye en Panamá, Colombia, Venezuela, Ecuador, Perú y Jamaica. En el Perú, solo está registrada $\mathrm{Ne}$ riacanthus lehmannianus (Lindau) Lindau (Leonard 1951, Brako y Zarucchi 1993, McDade et al. 2005). Carácter distintivo: Brácteas blancas a rosadas, corola regular a bilabiada, tubo corolino delgado, estambres 4 , estaminodio 1 , anteras monotecas y ausencia de cistolitos (Wasshausen 2013a).

21. Odontonema Nees nom. cons., Linnaea 16 (3): 300 (1842). Tipo: Justicia rubra Vahl (= Odontonema rubrum (Vahl) Kuntze.)

Género neotropical conformado por aproximadamente 30 especies de arbustos (McDade et al. 2000a). Se distribuye desde el norte de México hasta el sureste de Brasil, siendo comúnmente cultivado en zonas templadas del mundo (Daniel 1995). En el Perú, Odontonema cuspidatum (Nees) Kuntze, se encuentra cultivada en los jardines del departamento de Lima (Brako y Zarucchi 1993). Carácter distintivo: Corola infundibuliforme, limbo subactinomórfico a bilabiado, rojo a púrpura, estambres 2, anteras ditecas, estaminodios 2 y presencia de cistolitos (Wasshausen 2013a) (ver Figura 3F).

22. Oplonia Raf., Fl. Tellur. 4: 64 (1838). Tipo: Oplonia spinosa Raf.

Género tropical conformado por 14 especies de arbustos o pequeños árboles. Se distribuye en Argentina, las Antillas, Madagascar y el norte de Perú (Wasshausen y Ezcurra 1993, McDade et al. 2000a). En Perú se han registrado Oplonia grandiflora (Lindau) Stearn y Oplonia hutchisonii Wassh., siendo esta última endémica (León 2006). Carácter distintivo: Flores solitarias o en fascículos, axilares, corola débilmente bilabiada, estambres 2, estaminodios 2, anteras ditecas y presencia de cistolitos (Wasshausen y Ezcurra 1993).

23. Orophochilus Lindau., Bull. Herb. Boissier 5: 657. 1897. Tipo: Orophochilus stipulaceus Lindau.

Género neotropical monotípico conformado solo por la especie herbácea Orophochilus stipulaceus Lindau endémico de Perú, presente en el Bosque Muy Húmedo Pre-Montano (BMHP) del departamento de San Martín (León 2006). Carácter distintivo: Inflorescencia espiga laxa, cáliz pentalobado, lobos laciniados, corola bilabiada, estambres 4, anteras monotecas y ausencia de cistolitos (Lindau 1897).

24. Pachystachys Nees, Fl. Bras. 9:99 (1847). Tipo: Pachystachys riedeliana Nees

Género neotropical conformado por 18 especies de hierbas o plantas sufrutescentes. Se distribuye desde México y las Antillas hasta Perú, Bolivia, Argentina, Guyana Francesa y la Amazonia Brasileña (Wasshausen 1986, Côrtes 2013, Wasshausen 2013a). Presenta su mayor diversidad en la Amazonia del Perú con aproximadamente 16 especies (Brako y Zarucchi 1993, León 2006, Côrtes 2013). Carácter distintivo: Espiga terminal prominente, brácteas 


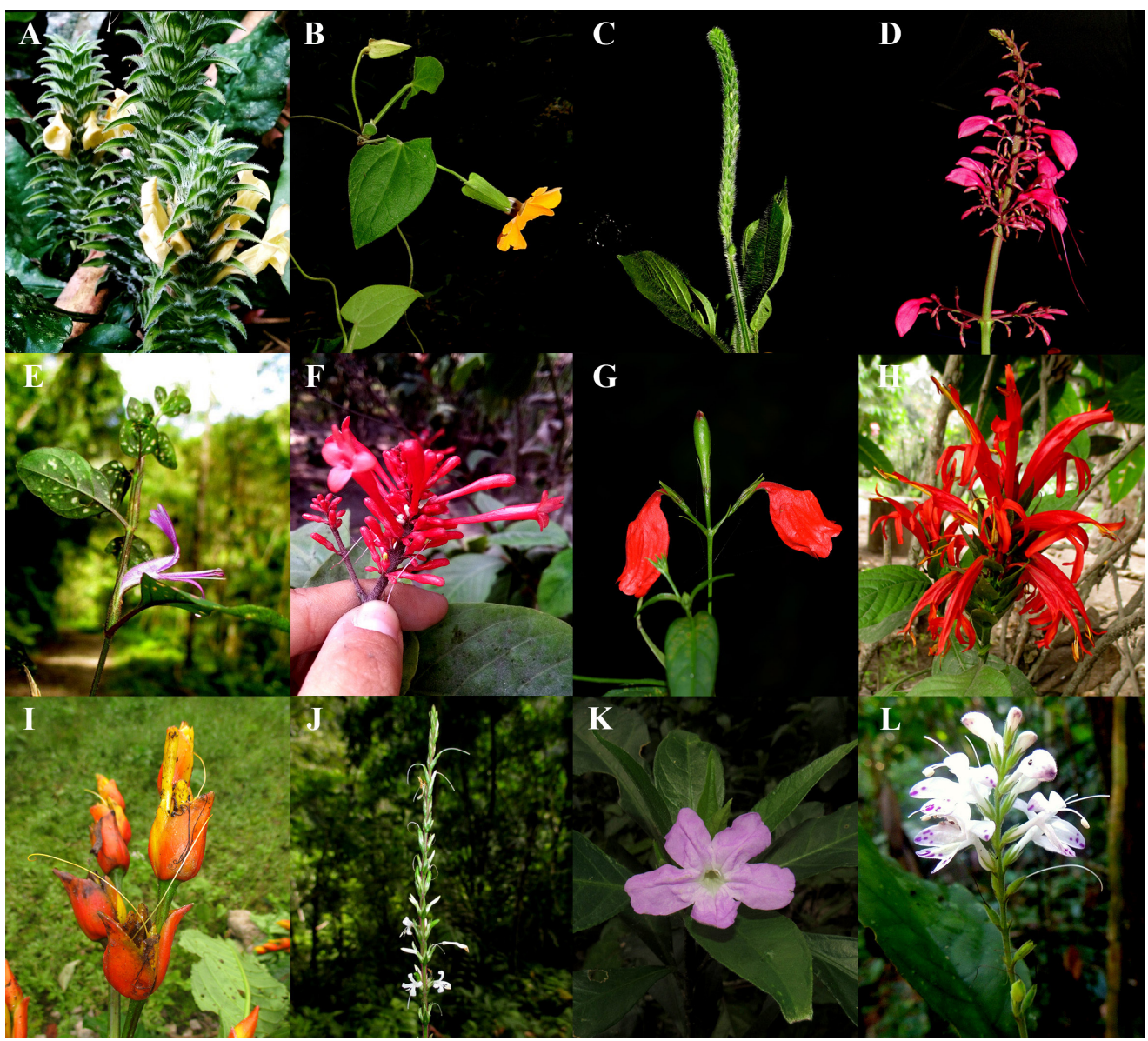

Figura 3. Especies de Acanthaceae presente en el Perú. A. Aphelandra pepe-parodii Wassh.; B. Thunbergia alata Bojer ex Sims; C. Fittonia sp.; D. Stenostephanus longistaminus (Ruiz \& Pav.) V.M. Baum; E. Hypoestes phyllostachya Baker; F. Odontonema cuspidatum (Nees) Kuntze; G. Ruellia brevifolia (Pohl) C. Ezcurra; H. Pachystachys spicata (Ruiz \& Pav.) Wassh.; I. Sanchezia oblonga Ruiz \& Pav.; J. Stenostephanus sprucei (Lindau) Wassh. \& J.R.I. Wood; K. Ruellia tarapotana Lindau; L. Pulchranthus adenostachyus (Lindau) V.M. Baum, Reveal \& Nowicke. Fotos por: A Florangel Condo; B-K Rosa Villanueva; L Mayra Briceño.

anchas foliosas o petaloides, corola bilabiada, tubo corolino ensanchándose hacia la base, estambres (2), tecas sagitadas y presencia de cistolitos (Wasshausen 1986, Côrtes 2013) (ver Figura $3 \mathrm{H})$.

25. Pseuderanthemum Radlk., Sitzungsber, Math.-Phys. Kl. Akad. Wiss. München 13: 282 (1883). Especie Lectotipo: Eranthemum alatum Nees (= Pseuderanthemum alatum (Nees) Radlk.).
Género tropical conformado por 60 especies de hierbas o arbustos. Ampliamente distribuido desde Asia, Australia, África a América (Choopan 2013, Wasshausen 2013a). En el Perú, se han reportado 7 especies (Brako y Zarucchi 1993, Wasshausen 1997a). Carácter distintivo: Corola pentalobada, estambres 2 , estaminodios 2 , cápsula con la parte fértil con un acúmen largo y presencia de cistolitos (Daniel 1993) (ver Figura 2B). 
26. Pulchranthus V.M. Baum, Reveal \& Nowicke, Syst. Bot. 8 (2): 212 (1983). Tipo: Odontonema surinamense Bremek. (= Pulchranthus surinamensis (Bremek.) V.M. Baum, Reveal \& Nowicke).

Género neotropical conformado por 4 especies de hierbas o subarbustos. Su distribución está restringida a la región Amazónica del norte de América del Sur, desde Colombia hasta Bolivia y Brasil. En el Perú, solo está registrada Pulchranthus adenostachyus (Lindau) Baum, Reveal \& Nowicke (Brako y Zarucchi 1993, Wasshausen 2013a). Carácter distintivo: Corola bilabiada, puntos oscuros en los lobos, tubo corolino corto, estambres 2 , anteras ditecas, estaminodios 2 y presencia de cistolitos (Wasshausen y Wood 2004) (ver Figura 3L).

27. Razisea Oerst., Vidensk. Meddel. Dansk Naturhist. Foren. Kjøbenhavn 1854 (812): 142 (1855). Tipo: Razisea spicata Oerst.

Género neotropical conformado por 5 especies de hierbas o arbustos. Se distribuye en Costa Rica, Panamá, Colombia, Ecuador y Perú, la mayor parte son endémicas para Costa Rica (Wasshausen 2013a). En el Perú, están registradas Razisea spicata Oerst. y Razisea ericae Mildbr. ex Wassh. (Brako y Zarucchi 1993, Wasshausen 1997b). Carácter distintivo: Corola cortamente bilabiada, labio superior entero, labio inferior ligeramente diferente del tubo de la corola, estambres 2, anteras monotecas y presencia de cistolitos (Wasshausen 1997b).

28. Ruellia L., Sp. Pl. 2: 634 (1753). Especie Lectotipo: Ruellia tuberosa L.

Género pantropical conformado por alrededor de 400 especies de hierbas perennes, arbustos, árboles pequeños o lianas (Wasshausen y Wood 2003, Tripp et al. 2013). El Neotrópico con aproximadamente 300 especies de Ruellia, es considerado su centro de diversificación (Ezcurra 1993; Tripp y Darbyshire 2017). En el Perú, están registrada 30 especies siendo RueIlia rauhii Wassh. endémica (Leonard 1951; Brako y Zarucchi 1993; Vásquez et al. 2002; León 2006; Wasshausen y Wood 2003, 2004; Wasshausen 2006, 2013a; McDade y Tripp 2007; Tripp y McDade 2012). Carácter distin- tivo: Corola pentalobada, débil a fuertemente zigomórfica, androceo con 4 estambres didínamos, anteras ditecas múticas y presencia de cistolitos (Tripp 2007) (ver Figura 3G y 3K).

29. Sanchezia Ruiz \& Pav., Fl. Peruv. Prodr. 5, t. 32 (1794). Especie Lectotipo: Sanchezia ovata Ruiz \& Pav.

Género neotropical conformado por alrededor de 56 especies de hierbas perennes o arbustos. Se distribuye en Sudamérica, Mesoamérica y el Caribe siendo Ecuador y Perú los centros de su diversificación (Wasshausen 2013a, Tripp y Koenemann 2015). En el Perú, se han registrado 48 especies siendo 8 de ellas endémicas (Brako y Zarucchi 1993, Wasshausen 1997a, Wasshausen y Wood 2004, León 2006, Wasshausen 2006). Carácter distintivo: Corola tubular con lobos retroflexos; estambres 2, un par de estaminodios, usualmente exertos, tecas mucronadas y presencia de cistolitos (Leonard 1926) (ver Figuras 2H y 3I).

30. Stenandrium Nees in Lindl., Nat. Syst. Bot., ed. 2: 444 (1836). Tipo: Stenandrium mandioccanum Nees.

Género pantropical conformado por alrededor de 60 a 70 especies de hierbas perennes caulescentes o no (McDade et al. 2005, Wasshausen 2013a). La mayor riqueza se encuentra en el Neotrópico, 40-50 especies están distribuidas desde el Sur de Estados Unidos hasta Argentina y Chile (Daniel 1984, McDade et al. 2005). En el Perú, están reportadas Stenandrium dulce (Cav.) Nees y Stenandrium mandioccanum Nees (Brako y Zarucchi 1993, Wasshausen 2013a). Carácter distintivo: Corola subactinomorfa a bilabiada, estambres 4, filamento corto, anteras monotecas y ausencia de cistolitos (Daniel 1984; Wasshausen 2013a).

31. Stenostephanus Nees in Mart., Fl. Bras. 9: 91 (1847). Tipo: Stenostephanus lobeliiformis Nees.

Género neotropical conformado por alrededor de 65 especies de hierbas o arbustos. Se distribuye desde el Oeste de México hasta Bolivia, siendo Colombia su principal centro de diversidad (Wasshausen 1999). En el Perú, 
se han registrado 7 especies, incluyendo 2 especies recientemente publicadas (Wasshausen 1999, 2006). Carácter distintivo: Inflorescencia racimo de dicasios o una panícula, corola bilabiada, androceo con 2 estambres, anteras monotecas y presencia de cistolitos (Wasshausen 1999) (ver Figura 3D y 3J).

32. Strobilanthes Blume, Bijdr. Fl. Ned. Ind. 781, 796 (1826). Especie Lectotipo: Strobilanthes cernua Blume

Género paleotropical conformado por 350 especies de hierbas y arbustos. Se distribuye principalmente en el Sureste de Asia (China e India) y Australia, siendo comúnmente cultivado en África y Centro América (Bennett y Scotland 2003, Moylan et al. 2004). En el Perú, solo está registrada la especie introducida Strobilanthes isophyllus (Nees) T. Anderson, cultivada en el departamento de Lima (Brako y Zarucchi 1993). Carácter distintivo: Corola infundibuliforme, lobos retusos, estambres 4 y presencia de cistolitos (Bennett y Scotland 2003).

33. Suessenguthia Merxm. Mitt. Bot. Staatssamml. München. 6: 178 (1953). Tipo: Suessenguthia trochilophila Merxm.

Género neotropical conformado por 10 especies arbustivas. Se distribuye en los Andes de Bolivia, el Sur y centro de Perú y el Oeste de Brasil (Schmidt-Lebuhn 2003). En el Perú, se han registrado 6 especies, siendo Suessenguthia vargasii var. hirsuta Schmidt-Leb. una variedad endémica (Brako y Zarucchi 1993; Schmidt-Lebuhn 2003; León 2006; Wasshausen 2006). Carácter distintivo: Brácteas conspicuas, inflorescencia de varias cabezuelas, corola pentalobada, estambres 4 y presencia de cistolitos (Schmidt-Lebuhn 2003).

34. Tessmaniacanthus Mildbr. Notizbl. Bot. Gart. Berlin-Dahlem 9: 987 (1926). Tipo: Tessmanniacanthus chlamydocardioides Mildbr.

Género neotropical monotípico conformado por la especie Tessmanniacanthus chlamydocardioides Mildbr. Se distribuye solo en el Perú, registrada en el departamento de Amazonas (Brako y Zarucchi 1993). Carácter distintivo:
Cáliz con lobos lineales, subiguales, corola bilabiada, estambres 2, anteras ditecas y presencia de cistolitos (Mildbraed 1926).

35. Tetramerium Nees in Benth. Bot. Voy. Sulphur. 48 (1846). Especie lectotipo: Tetramerium polystachyum Nees.

Género neotropical conformado por 29 especies de hierbas o subarbustos (Daniel et al. 2008). Se distribuye desde el Sur de Estados Unidos hacia México, América Central y el Sur de América del Sur (Venezuela, Colombia, Perú, Ecuador y Bolivia) (Daniel 1986). En el Perú, se han registrado 7 especies siendo 5 endémicas (Brako y Zarucchi 1993, León 2006). Carácter distintivo: Brácteas de la inflorescencia cuadrangulares, corola bilabiada, estambres 2, anteras ditecas y presencia de cistolitos (Daniel y Acosta 2003, Wasshausen 2013a) (ver Figura 2G).

36. Thunbergia Retz, Physiogr. Salsk. Handl. 1(3): 163 (1780). Tipo: Thunbergia capensis Retz.

Género paleotropical conformado por alrededor de 90 especies de hierbas perennes, erectas a extendidas, arbustos o enredaderas (Wasshausen 2013a). Se distribuye en las regiones tropicales y subtropicales de Asia, África, Madagascar y Australia siendo 5 especies ampliamente cultivadas en el Neotrópico (Retief y Reyneke 1984; Borg et al 2008). En el Perú, están reportadas Thunbergia fragans Roxb., Thunbergia laurifolia Lindl. y Thunbergia alata Retz., especies naturalizadas en el país. Carácter distintivo: Brácteolas (2) grandes que envuelven el cáliz, corola infundibuliforme pentalobada, estambres (4) didínamos, lobos estigmáticos desiguales, cápsula globosa sin retináculo y ausencia de cistolitos (Retief y Reyneke 1984, Schönenberger 1999) (ver Figura 3B).

37. Trichanthera Kunth, Nov. Gen. Sp. (folio ed.) 2: 243, in obs. (1818). Tipo: Ruellia gigantea Bonpl. (=Trichanthera gigantea (Bonpl.) Nees).

Género neotropical conformado por 2 especies de arbustos o árboles. Se distribuye desde América Central hacia Ecuador, Brasil y Perú. 
En el Perú, solo está registrado Trichanthera gigantea (Bonpl.) Nees en un rango altitudinal entre los 0-1000 msnm (Brako y Zarucchi 1993, Wasshausen 2013a). Carácter distintivo: Cáliz tomentoso, corola pentalobado, estambres 4 , didínamos, exertos, anteras ditecas, tecas espolonadas y presencia de cistolitos (Wasshausen 2013a).

38. Trichosanchezia Mildbr., Notizbl. Bot. Gart. Berlin-Dahlem 9: 984 (1926). Tipo: Trichosanchezia chrysothrix Mildbr.

Género tropical monotípico conformado por la especie herbácea Trichosanchezia chrysothrix Mildbr. endémica de Perú, se distribuye en el Nor-oriente específicamente en la cuenca del Santiago y del Imaza en el departamento de Amazonas (León 2006). Carácter distintivo: Cáliz pentalobado, lobos largos, densamente hirsuto, corola tubular, tubo corolino muy largo, estambres 4 , anteras ditecas y presencia de cistolitos (Mildbraed 1926).

\section{Conclusiones}

El presente trabajo es una puesta al dia del conocimiento taxonómico de la familia Acanthaceae, importante en la flora de Perú por su riqueza de especies y su valor ecológico y ornamental. Se actualiza a 314 el número de especies, agrupándose en 38 géneros siendo Justicia (64) y Aphelandra (50) los más ricos en especies. Asimismo, Cephalacanthus, Orophochilus y Trichosanchezia son géneros monotípicos endémicos. $Y$ se adicionan 3 especies endémicas para el país, Stenostephanus macrolobus (Lindau) J.R.I. Wood, Elytraria klugii Leonard y Pachystachys linearibracteata A.L.A. Côrtes. Esta familia requiere de revisiones taxonómicas de sus géneros, no encontrándose información actualizada en muchos de ellos. Por lo tanto, la continuidad en las colectas botánicas, inventarios florísticos y estudios taxonómicos son de gran importancia ya que permitirán una mejor comprensión de la diversidad de especies, estados de conservación, rangos de distribución, endemismos e importancia biológica que representa la familia Acanthaceae en la flora peruana.

\section{Agradecimientos}

La primera autora agradece al Dr. Tom Daniel y a la Dra Erin Tripp por sus valiosos comentarios sobre las especies actualmente reportadas para Perú. Además, se agradece a Robin Fernandez Hilario y Mayra Briceño Huayta por compartir fotos de sus colectas en campo.

\section{Bibliografía}

Al-Hakimi, A.; Maideen, H.; Latiff, A. 2015. Pollen and Seed Morphology of Rhinacanthus Nees and Hypoestes Sol. ex R. Br. (Acanthaceae) of Yemen. Sains Malaysiana 44(1): 7-15.

APG IV. 2016. An update of the Angiosperm Phylogeny Group classification for the orders and families of flowering plants: APG IV. Botanical Journal of the Linnean Society 181: $1-20$.

Balkwill, MJ.; Balkwill, K. 1997. Delimitation and Infra-generic Clasification of Barleria (Acanthaceae). Kew Bulletin 52(3): 535-573.

Bennett, J.; Scotland, R. 2003. A revision of Strobilanthes (Acanthaceae) in Java. Kew Bulletin 58(1):1-82.

Bentham, G. 1876. Acanthaceae. In Bentham, G.; Hooker, J. (eds.) Genera Plantarum. London, UK, Reeve .v. 2, pt. 2, p. 1060-1122.

Boggan, J.; Funk, V.; Kellof, C. 1997. Checklist of the Plants of the Guianas (Guyana, Surinam, Franch Guiana), 2 ed. Washinsgton, Estados Unidos, Museum of Natural History, Smithsonian Institution. $238 \mathrm{p}$.

Borg, J.; McDade, L.; Schonenberger, J. 2008. Molecular phylogenetics and Morphological evolution of Thunbergioideae (Acanthaceae). Taxon 57(3): 811-822.

Brako, L.; Zarucchi, J. 1993. Catálogo de las Angiospermas y Gimnospermas del Perú. Monog. Syst. Bot. Missouri Bot. Gard. 45: 1-1286.

Braz, DM; Monteiro, R. 2017. Taxonomic Revision of Staurogyne (Nelsonioideae, Acanthaceae) in the Neotropics. Phytotaxa 296(1):1-40. 
Bremekamp, C. 1965. Delimitation and subdivision of the Acanthaceae. Bulletin of the Botanical Survey of India 7: 21-30.

Choopan, T. 2013. Systematic study of Acanthaceae, subfamilies Nelsonioideae and Acanthoideae (Pseuderanthemum), in Thailand. Tesis PhD. THA, Suranaree University of Technology. $478 \mathrm{p}$.

Chumchin, N.; McDade, L.; Fisher, A. 2015. Phylogeny of Dyschoriste (Acanthaceae). Aliso 33(2): 77-89.

Clarke, C. 1885. Acanthaceae. In Hooker, J. (ed.). Flora of British India. London, UK, Reeve. v. 4, p. 387-558.

Côrtes, AL. 2013. Sistemática e biogeografía (Acanthaceae) da linhagem Tetramerium na América do Sul. Tesis Dr. Estado de Bahia, Brasil, Universidade Estadual de Feira de Santana. $172 \mathrm{p}$.

Cronquist, A. 1988. The evolution and classification of flowering plants. 2 ed. Bronx, US, The New York Botanical Garden. 555 p.

Daniel, T. 1984. A Revision of Stenandrium (Acanthaceae) in Mexico and Adjacent Regions. Ann. Missouri Bot. Gard. 71: 1028-1043.

Daniel, T. 1986. Systematics of Tetramerium (Acanthaceae). Syst. Bot. Monogr. 12: 1-134.

Daniel, T. 1988. A systematic study of Bravaisia DC. (Acanthaceae). Proc. Calif. Acad. Sci. 45(8): 111-132.

Daniel, T. 1991. A revision of Aphelandra (Acanthaceae) in Mexico. Proc. Calif Acad Sci. 47:235-274.

Daniel, T. 1993. Taxonomic and geographic notes on Central American Acanthaceae. Contr. Univ. Michigan Herb. 48(5): 119-130.

Daniel, T. 1995. Revision of Odontonema (Acanthaceae) in Mexico. Contr. Univ. Michigan Herb. 20: 147-171.

Daniel, T. 2004. Acanthaceae of Sonora: taxonomy and phytogeography. Proceedings of the California Academy of Sciences 55: 690805 .
Daniel, T. 2009. Synopsis of Dicliptera (Acanthaceae) in the Nueva Galicia region of western Mexico with a new species, D. novogaliciana. Proc. Calif. Acad. Sci. 60: 1-18

Daniel, T. 2016. Avicennia (Acanthaceae: Avicennioideae) in North America and Mesoamerica. Proceedings of the California Academy of Sciences 63 (5): 163-189.

Daniel, T.; Acosta, S. 2003. Acanthaceae. Flora del Bajío y de regiones adyacentes 117: 1- 177.

Daniel, T.; McDade, L.; Manktelow, M; Kiel, C. 2008. The "Tetramerium Lineage" (Acanthaceae: Acanthoideae: Justicieae): Delimitation and Intra-Lineage Relationships Based on $\mathrm{cp}$ and nrITS Sequence Data. Systematic Botany 33 (2): 416-436.

Daniel, T.; McDade, L. 2014. Nelsonioideae (Lamiales: Acanthaceae): Revision of Genera and Catalog of Species. Aliso: A Journal of Systematic and Evolutionary Botany 32 (1):1-45.

Daniel, T.; Lott, E. 2016. Acantáceas (Acanthaceae). In CONABIO (ed.). La Biodiversidad en Colima. Estudio de Estado. México. p. 229-237.

Darbyshire, I. 2015. The genus Hypoestes (Acanthaceae) in Angola. Kew Bulletin 70:44.

Durkee, L. 1978. Acanthaceae. In Woodson, R.; Scherry, R. (eds.). Flora of Panama. Ann. Missouri Bot. Gard. 65(1): 155-283.

Durkee, L. 1986. Flora Costaricensis (en línea). Fieldiana 18: 1-96. Consultado ene-feb 2019. Disponible en https://archive.org/details/floracostaricens40burg

Engler, A.; Diels, L. 1936. Syllabus der Pflanzenfamilien. Berlin, GE. v. 11.

Ezcurra, C. 1993. Systematics of Ruellia (Acanthaceae) in southern South America. Ann. Missouri Bot. Gard. 80:787-845.

Ezcurra, C. 2002. El género Justicia (Acanthaceae) en Sudamérica Austral. Ann. Missouri Bot. Gard. 89 (2): 225.

Ezcurra, C. 2014. Estudio Taxonómico del género Dyschoriste (Acanthaceae) en la Argentina. Darwiniana 2(2): 22-236. 
Gentry, A. 1993. A Field Guide to the Families and Genera of Woody Plants of Northwest South America (Colombia, Ecuador, Peru) with supplementary notes on herbaceous taxa. Washington, US, Conservation International. $895 \mathrm{p}$.

Gibson, D. 1974. Acanthaceae. In Standley, P. (ed.). Flora of Guatemala. Fieldiana 24 (10): 328-461.

Graham, V. 1988. Delimitation and infra-generic classification of Justicia (Acanthaceae). Kew Bull. 34: 351-624.

Hutchinson, J. 1973. The families of flowering plants arranged according to a new system based on their probable phylogeny. 3 ed. Oxford, UK, Clarendon Press. 968p.

Indriunas, A. 2011. Revisão taxonômica de Herpetacanthus Nees (Acanthaceae). Tesis Mag. São Paulo, Brasil, Instituto de Botânica.

Indriunas, A.; Kameyama, C. 2012. New Species of Herpetacanthus (Acanthaceae) from the Atlantic Forest and Neighboring Areas (Brazil). Systematic Botany 37(4):1006-1022.

Jussieu, A. 1789. Ordo Acanthi. Genera Plantarum. Paris, FR, s. e. 498 p.

Kameyama, C. 2008. New species, nomenclatural changes and lectotypifications in Neotropical Lepidagathis Willd. (Acanthaceae). Kew Bulletin, 63: 565 - 581.

Kiel, C.; McDade, L.; Daniel, T.; Champluvier, D. 2006. Phylogenetic Delimitation of Isoglossinae (Acanthaceae: Justicieae) and Relationships among Constituent Genera. Taxon 55(3):683-694.

Kiel, C., Daniel, T.; Darbyshire, I.; McDade, L. 2017. Unraveling relationships in the morphologically diverse and taxonomically challenging 'justicioid' lineage (Acanthaceae, Justicieae). Taxon 66: 645-674.

Kiel, C., Daniel, T.; McDade, L. 2018. Phylogenetics of New World 'justicioids' (Justicieae: Acanthaceae): Major Lineages, Morphological Patterns, and Widespread Incongruence with Classification. Systematic Botany 43(2):459-484.
Leonard, E. 1926. Notes on the genus Sanchezia. J. Wash. Acad. Sci. 16: 484-492.

Leonard, E. 1951'. The Acanthaceae of Colombia, I (en línea): subfamilies Nelsonioideae, Mendoncioideae, Thunbergioideae, Acanthoideae. Contr. U.S. Natl. Herb. 31(1-3): 1-117. Consultado ene - feb. 2019. Disponible en https://archive.org/details/cbarchive101324acanthaceaeofcolombia1958

Leonard, E. 1953. The Acanthaceae of Colombia, II: Subfamily Acanthoideae, continued. Contr. U.S. Natl. Herb. 31 (2): 119-322.

Leonard, E. 1958. The Acanthaceae of Colombia, III: Subfamily Acanthoideae completed. Contr. U.S. Natl. Herb. 31(3): 323-781.

León, B. 2006. Acanthaceae endémicas del Perú (en línea). Revista peruana de Biología 13(2):23-29. Consultado ene-feb 2019. Disponible en http://www.scielo.org.pe/pdf/rpb/ v13n2/v13n02a005.pdf

Lindau, G. 1895. Acanthaceae. In Engler, A.; Prantl, K. (eds.). Die Natürlichen Pflanzenfamilien. Leipzig, DE, s. e. teil 4, abt. 3b, p. 274-354.

Lindau, G. 1897. Acanthaceae Americanae et Asiaticae: Novae Vel Minus cognitae. Bull. Herb. Boissier 5:657-658.

Lindau, G. 1905. Plantae nonnullae novae andinae. Repert. Spec. Nov. Regni Veg. 1: 158.

Linnaeus, C. 1753a. Species Plantarum. SUE, s.e. tomo $1,560 \mathrm{p}$.

Linnaeus, C. 1753b. Species Plantarum. SUE, s.e. tomo 2, 561-1200 p.

Macbride, J. F. 1936. Rubiaceae. En Flora of Peru. Field Mus. Nat. Hist., Bot. Ser. 13(6/1): 3-261

Macbride, J. F. 1962. Solanaceae. En J.F. Macbride, Flora of Peru. Field Mus. Nat. hist., Bot. Ser. 13(Part V-B, 1): 3-267.

Martín-Bravo, S.; Daniel, T. 2016. Molecular evidence supports ancient long-distance dispersal for the amphi-Atlantic disjunction in the giant yellow shrimp plant (Barleria oenotheroides). American Journal of Botany 103(6): 1103-1116. 
McDade, L. 1984. Systematics and Reproductive Biology of the Central American Species of the Aphelandra pulcherrima Complex (Acanthaceae). Ann. Missouri Bot. Gard. 71(1): 10416.

McDade, L.; Daniel, T.; Masta, S.; Riley, K. 2000a. Phylogenetic Relationships within the Tribe Justicieae (Acanthaceae): Evidence from Molecular Sequences, Morphology, and Cytology. Ann. Missouri Bot. Gard. 87(4): 435-458.

McDade, L.; Masta, S.; Moody, M.; Waters, E. 2000b. Phylogenetic relationships among Acanthaceae: Evidence from two genomes. Systematic Botany 25: 106-121.

McDade, L.; Daniel, T.; Kiel, C.; Vollesen, R. 2005. Phylogenetic Relationships among Acantheae (Acanthaceae): Major Lineages Present Contrasting Patterns of Molecular Evolution and Morphological Differentiation. Systematic Botany 30(4):834-862.

McDade, L.; Tripp, E. 2007. A Synopsis of Costa Rican Ruellia (Acanthaceae), with Descriptions of Four New Species. Brittonia 59 (3): 199-216.

McDade, L.; Daniel, T.; Kiel, C. 2008. Toward a Comprehensive Understanding of Phylogenetic Relationships among Acanthaceae s.l. (Lamiales). American Journal of Botany 95: 1136-1152.

McDade, L.; Daniel, T.; Kiel, A.; Borg, J. 2012. Phylogenetic placement, delimitation, and relationships among genera of the enigmatic Nelsonioideae (Lamiales: Acanthaceae). Taxon 61 (3): 637-651.

Melchior, H. 1964. Engler's syllabus der Pflanzenfamilien. Berlin, Alemania.

Mildbraed, J. 1926. Plantae Tessmannianae Peruvianae III. Notizbl. Bot. Gart. Berlin-Dahlem 9(89):964-997.

Moylan, E.; Bennett, J.; Carine, M.; Olmstead, R.; Scotland, R. 2004. Phylogenetic relationships among Strobilanthes SL (Acanthaceae): Evidence from ITS nrDNA, trnL-F cpDNA, and morphology. American Journal of Botany 91 (5): 724-735.
Nees von Esenbeck, C. 1847. Acanthaceae. In De Candolle, A. (ed.). Prodromus Systematis Naturalis Regni Vegetabilis. París, FR, Treuttel \& Würtz. v. 11, p. 46- 519.

Olmstead, R.; Bremer, B.; Scott, K.; Palmer, J. 1993. A parsimony analysis of the Asteridae sensu lato based on rbcL sequences. Ann. Missouri Bot. Gard. 80(3): 700-722.

Reveal, J. 2012. An outline of a classification scheme for extant flowering plants. Phytoneruron 37: 1-221.

Retief, E.; Reyneke, W. 1984. The genus Thunbergia Retz in southern Africa. Bothalia 15(12): $107-116$.

Schmidt-Lebuhn, A. 2003. A taxonomic revision of the genus Suessenguthia Merxm. (Acanthaceae). Candollea 58(1): 101-128.

Schmidt-Lebuhn, A.; Schwerdtfeger, M.; Kessler, M.; Lohaus, G. 2007. Phylogenetic constraints vs. ecology in the nectar composition of Acanthaceae. Flora 202: 62-69.

Schwarzbach, A.; McDade, L. 2002. Phylogenetic Relationship of the Mangrove Family

Avicenniaceae based on chloroplast and nuclear ribosomal DNA sequences. Systematic Botany 27(1): 84-98.

Scotland, R.; Sweere, J.; Reeves, P.; Olmstead, R. 1995. Higher-level systematics of Acanthaceae determined by chloroplast DNA sequences. American Journal of Botany 82: 266-275.

Scotland, R.; Vollesen, K. 2000. Classification of Acanthaceae. Kew Bulletin 55: 513-589.

Schönenberger, J.; Endress, P. 1998. Structure and Development of the Flowers in Mendoncia, Pseudocalyx, and Thunbergia (Acanthaceae) and Their Systematic Implications. International Journal of Plant Sciences 159 (3):4446-465.

Schönenberg, J. 1999. Floral structure, development and diversity in Thunbergia (Acanthaceae). Botanical Journal of the Linnean Society 130:1-36.

Singh, G. 2004. Plant systematics. An integrated approach: Acanthaceae. New Hampshire, US, Science Publisher. p. 494-496. 
Soto, A.; Vega, G. 2010. Plantas con flores que atraen mariposas. Santo Domingo de Heredia, Costa Rica, Instituto Nacional de Biodiversidad (INBio). p. 14-15.

Stevens, P. F. 2017. Angiosperm Phylogeny Website. Consultado ene 2019. Disponible en http://www.mobot.org/MOBOT/research/APweb/

Takhtajan, A. 1987. Systema magnoliophytorum. Leningrand, RU, Nauka. 438 p.

Takhtajan, A. 2009. Flowering plants, 2 ed. Heidelberg, GE, Science \& Business Media.871 p.

Thorne, R. 1992. An updated classification of the flowering plants. Aliso 13: 365-389.

Thorne, R. 2007. An updated classification of the Class Magnoliopsida ("Angiospermae").

Tomlinson, P. 1986. The botany of mangroves. Cambridge, UK, Cambridge University Press. 413 p. The Botanical Review 73(2): 67-182.

Tripp, E. 2007. Evolutionary relationships within the species-rich genus Ruellia (Acanthaceae). Syst. Bot 32: 628-649.

Tripp, E.; Fatimah, S. 2012. Comparative anatomy and morphology of the African genus $\mathrm{Sa}$ tanocrater (Acanthaceae). Am. J. Bot. 99:967982.

Tripp, E.; McDade, L. 2012. New synonymies for Ruellia (Acanthaceae) of Costa Rica and notes on other Neotropical species. Brittonia 64 (3): 305-317.

Tripp, E.; Daniel, T.; Fatimah, S.; McDade, L. 2013. Phylogenetic Relationships within Ruellieae (Acanthaceae) and a Revised Classification. Int. J. Plant Sci. 174: 97-137.

Tripp, E.; McDade, L. 2014. A Rich Fossil Record Yields Calibrated Phylogeny for Acanthaceae (Lamiales) and Evidence for Marked Biases in Timing and Directionality of Intercontinental Disjunctions. Syst. Biol. 63(5): 660-684.

Tripp, E.; Koenemann, D. 2015. Nomenclatural Synopsis of Sanchezia (Acanthaceae), Fifty Years Since Last Treated. Novon 24: 213-221.
Tripp, E.; Darbyshire, I. 2017. Phylogenetic Relationships among Old World Ruellia L.: A New Classification and Reinstatement of the Genus Dinteracanthus Schinz. Systematic Botany 42 (3): 470-483.

Tripp, E.; Luján, M. 2018. Venezuelan Ruellia (Acanthaceae) A Monograph. Memoirs of the New York Botanical Garden 119:1-75.

Ulloa Ulloa, C.; Zarucchi, J.; León, B. 2004. Diez años de adiciones a la flora del Perú: 19932003. Arnaldoa Ed. Especial: 7-242.

Van Tieghem, M. 1908. Structure du pistil et de l'ovule du fruit et de la graine des Acanthacées. Annales des Science Naturelles Botanique (Serie 9) 7:1-24.

Vásquez, R.; Rojas, R.; Rodríguez, E. 2002. Adiciones a la Flora Peruana: especies nuevas, nuevos registros y estados taxonómicos de las Angiospermas para el Perú. Arnaldoa 9 (2): 43-110.

Vollesen, K. 2008. Acanthaceae (part 1). In Beentje, H.; Ghazanfar, S. (eds.). Flora of Tropical East Africa. Royal Botanic Gardens, Kew. $286 \mathrm{p}$.

Wasshausen, D. 1975. The genus Aphelandra (Acantheceae). Smithsonian Contr. Bot. 18: $1-157$.

Wasshausen, D. 1986. The Systematics of the Genus Pachystachys (Acanthaceae). Proc. Biol. Soc. Wash. 99(1): 160-185.

Wasshausen, D. 1996. New species and new combinations in Aphelandra (Acanthaceae) from Ecuador and adjacent Peru. Nord. J. Bot. 16: 389-407.

Wasshausen, D. 1997a. A checklist of the Acanthaceae collected by John J. Wurdack in Amazonian Peru. BioLlania Ed. Especial 6: 541550 .

Wasshausen, D. 1997b. Razisea ericae (Acanthaceae), a New Species from Ecuador and Adjacent Peru. Novon 7:88-91.

Wasshausen, D. 1999. The genus Stenostephanus (Acanthaceae) in Bolivia. Harvard Papers in Botany 4(1): 279-288. 
Wasshausen, D. 2006. A checklist of the Acanthaceae collected in the "Sira mountains" of Peru. Ann. Naturhistorisches Museum Wien.108 B: 167-190.

Wasshausen, D. 2013a. Acanthaceae. In Persson, C.; Stahl, B. (eds.) Flora of Ecuador. Goteborg, Sweden, University of Gothenburg. 328 p.

Wasshausen, D. 2013b. New species of Aphelandra (Acanthaceae) from Peru and Ecuador. Journal of the Botanical Research Institute of Texas 7(1): 109-120.

Wasshausen, D. 2017. Mendoncia. In Acevedo, R. (ed.). Guide to the genera of lianas and climbing plants in the Neotropics. Acanthaceae. Washington, Estados Unidos, Smithsonian Institution.

Wasshausen, D., Ezcurra, C. 1993. A New Species of Oplonia (Acanthaceae) from Southern South America. Novon 3(1): 89-91.

Wasshausen, D.; Wood, J. 2001. Further discoveries in the Genus Stenostephanus (Acanthaceae) in Bolivia. Harvard Papers in Botany 6 (2): 449-454.

Wasshausen, D.; Wood, J. 2003. Notes on the Genus Justicia in Bolivia. Kew Bulletin 58(4):769-831.

Wasshausen, D.; Wood, J. 2004. Acanthaceae of Bolivia (en línea). Contribution from the United States National Herbarium 49: 1-152. Consultado ene - feb. 2019. Disponible en https:// botany.si.edu/pubs/CUSNH/vol49 web.pdf

Wenk, R.; Daniel, T. 2009. Molecular phylogeny of Nelsonioideae (Acanthaceae) and phylogeography of Elytraria. Proc. Calif. Acad. Sci. 60: 53-68.

Wettstein, R. 1924. Handbuch der systematischen Botanik. Leipzig, GE. 943 p.

Wood, J. 2009. New names, combinations and synonyms in Justicia and Stenostephanus (Acanthaceae). Kew Bulletin 64 (1): 49-55. 